Proceedings of the Edinburgh Mathematical Society (2006) 49, 427-433 (C)

DOI:10.1017/S0013091504001403 Printed in the United Kingdom

\title{
EXTENDING A RESULT OF RYAN ON WEAKLY COMPACT OPERATORS
}

\author{
KAZUYUKI SAITÔ ${ }^{1}$ AND J. D. MAITLAND WRIGHT ${ }^{2}$ \\ ${ }^{1}$ Mathematical Institute, Tôhoku University, Aoba, Sendai 980-8578, Japan \\ ${ }^{2}$ Mathematical Sciences, King's College, \\ University of Aberdeen, Aberdeen AB24 3UE, UK \\ (jdmw@maths.abdn.ac.uk; yk.saito@beige.plala.or.jp)
}

(Received 27 November 2004)

\begin{abstract}
An elegant result of Ryan gives a characterization of weakly compact operators from a Banach space $A$ into $c_{0}(X)$, the space of null sequences in a Banach space $X$. It would be a useful tool if the analogue of Ryan's result were valid when $c_{0}(X)$ is replaced by $c(X)$, the space of convergent sequences in $X$. This seems plausible and has been assumed to be true by some authors. Unfortunately, it is false in general; Ylinen has produced a counterexample. But when $A$ is a $C^{*}$-algebra, or, more generally, when the dual of $A$ is weakly sequentially complete, we show that the desired extension of Ryan's result does hold. The latter result turns out to be 'best possible'.
\end{abstract}

Keywords: weakly compact; weakly sequentially complete; $C^{*}$-algebras

2000 Mathematics subject classification: Primary 46B99

\section{Introduction}

The origin of this paper stems from observing that some results on non-commutative, finitely additive vector measures (i.e. weakly compact operators from a $C^{*}$-algebra to a Banach space) do not depend on the domain being a $C^{*}$-algebra but are essentially Banach space results.

Let $A$ and $X$ be Banach spaces and let $\left(T_{n}\right)(n=1,2, \ldots)$ be a sequence of weakly compact operators mapping $A$ into $X$. For each $z \in A^{* *}$ let $\left(T_{n}^{* *} z\right)(n=1,2, \ldots)$ be a Cauchy sequence. Since, for each $n, T_{n}$ is weakly compact, the range of $T_{n}^{* *}$ is in $X$. By the uniform boundedness theorem there is a bounded operator $T^{\#}: A^{* *} \mapsto X$ such that $\lim _{n \rightarrow \infty} T_{n}^{* *} z=T^{\#} z$ for each $z$ in $A^{* *}$. It would be natural to expect $T^{\#}$ to be weakly compact but, in general, this is false. This follows from the following example constructed by Ylinen $[6]$.

In [6, Proposition 2.1], $A=l^{1}=X$. For each $n, T_{n}: l^{1} \mapsto l^{1}$ is defined by

$$
T_{n}\left(x_{1}, x_{2}, \ldots, x_{k}, \ldots\right)=\left(x_{1}, x_{2}, \ldots, x_{n}, 0,0, \ldots\right) .
$$


Then each $T_{n}$ is weakly compact (because its range is finite dimensional). Ylinen proves that $\left(T_{n}^{* *} z\right)(n=1,2, \ldots)$ converges for each $z$ in the dual of $l^{\infty}$ but the pointwise limit of the sequence of operators $\left(T_{n}\right)(n=1,2, \ldots)$ is not weakly compact.

However, if $A$ is a $C^{*}$-algebra, then there does exist a weakly compact operator $T: A \mapsto$ $X$ such that $\lim _{n \rightarrow \infty} T_{n}^{* *} z=T^{* *} z$ for each $z$ in $A^{* *}$. This is an immediate consequence of [1, Corollary 3.3]. In this note we show that a positive result is also obtained if $A^{*}$ is weakly complete. (We recall that the dual of a $C^{*}$-algebra is always weakly complete.) We shall also see that, in a sense made precise here, the latter result is 'best possible'.

Ryan [4] characterized weakly compact operators from a Banach space $A$ into $c_{0}(X)$, the space of null sequences in a Banach space $X$ (see Proposition 3.4 below). When $c_{0}(X)$ is replaced by $c(X)$, the space of convergent sequences in $X$, the natural extension of Ryan's characterization does not hold, in general. But when $X^{*}$ is weakly (sequentially) complete, then we show, in $\S 3$, that Ryan's characterization can be generalized successfully by applying the results we obtain in $\S 2$. This can then be applied to underpin some fundamental work on weak compactness and multilinear operators on Banach spaces [3].

\section{Convergent sequences of weakly compact operators}

Let us recall that a Banach space $Z$ is said to be weakly complete if, whenever $\left(z_{n}\right)$ $(n=1,2, \ldots)$ is a sequence in $Z$ such that $\left(\phi z_{n}\right)(n=1,2, \ldots)$ is a Cauchy sequence for every $\phi$ in $Z^{*}$, then there exists $z$ in $Z$ such that $\phi z_{n} \rightarrow \phi z$ for every $\phi$ in $Z^{*}$. Some authors use the term weakly sequentially complete for the same property.

Theorem 2.1. Let $A$ be a Banach space such that $A^{*}$ is weakly complete. Let $X$ be a Banach space and let $\left(T_{n}\right)(n=1,2, \ldots)$ be a sequence of weakly compact operators from $A$ into $X$. Let $\left(T_{n}^{* *} z\right)(n=1,2, \ldots)$ be a Cauchy sequence for each $z$ in $A^{* *}$. Then there exists a weakly compact operator $T$ such that $\left\|\left(T^{* *}-T_{n}^{* *}\right) z\right\| \rightarrow 0$ for each $z$ in $A^{* *}$.

Proof. Since $T_{n}$ is weakly compact, $T_{n}^{* *}$ maps $A^{* *}$ into $X$. Let $T^{\#} z=\lim T_{n}^{* *} z$ for each $z$ in $A^{* *}$. Then, by the uniform boundedness theorem, $T^{\#}$ is a bounded linear operator from $A^{* *}$ into $X$. Let $T$ be the restriction of $T^{\#}$ to $A$.

Fix $\phi \in X^{*}$. Then, for each $z \in A^{* *}$,

$$
\lim _{n \rightarrow \infty}\left\langle T_{n}^{* *} z, \phi\right\rangle=\left\langle T^{\#} z, \phi\right\rangle .
$$

So

$$
\lim _{n \rightarrow \infty}\left\langle z, T_{n}^{*} \phi\right\rangle=\left\langle T^{\#} z, \phi\right\rangle
$$

So $\left(T_{n}^{*} \phi\right)(n=1,2, \ldots)$ is a weakly Cauchy sequence in $A^{*}$. By the hypothesis that $A^{*}$ is weakly complete, it follows that there exists a unique $\alpha \in A^{*}$ such that $\langle z, \alpha\rangle=\left\langle T^{\#} z, \phi\right\rangle$ for all $z$ in $A^{* *}$.

All that is now needed is to show that $T^{* *}=T^{\#}$. Since this has been a source of error in the past we wish to avoid being too glib and so give a detailed elementary argument.

Let $\left(z_{t}\right)$ be a net in $A^{* *}$ which converges to 0 in the $\sigma\left(A^{* *}, A^{*}\right)$-topology. So $\left\langle z_{t}, \alpha\right\rangle \rightarrow 0$. Thus $\left\langle T^{\#} z_{t}, \phi\right\rangle \rightarrow 0$ for each $\phi$ in $X^{*}$. So $T^{\#}$ is a continuous map of $A^{* *}$, equipped with 
the weak*-topology, to $X$ equipped with the weak topology. Since the norm closed unit ball of $A^{* *}$ is weak* compact, the image of the unit ball of $A^{* *}$ under the map $T^{\#}$ is weakly compact. Hence $T^{\#}$, and its restriction to $A, T$, is weakly compact. Thus, by Lemma VI.2.3 and Theorem VI.4.2 of [2], $T^{* *}$ is weak* to weak continuous from $A^{* *}$ to $X$. By Goldstine's theorem (see [2, Theorem V.4.5]), the norm closed unit ball of $A$ is weak*-dense in the norm closed unit ball of $A^{* *}$. Hence $T^{\#}=T^{* *}$.

Remark. Let $A$ be a $C^{*}$-algebra. Its dual is then the predual of a von Neumann algebra and so, by [5, Corollary III.5.2], the dual of $A$ is weakly complete. Hence Theorem 2.1 applies whenever $A$ is a $C^{*}$-algebra.

It turns out that Theorem 2.1 is 'best possible'. To make this claim precise it is convenient to introduce the following definition.

Definition 2.2. Let $X$ be a Banach space. A Banach space $A$ is said to have the weak compactness stability property with respect to $X$ if, given any sequence of weakly compact operators $\left(T_{n}\right)(n=1,2, \ldots)$, each mapping $A$ into $X$, and with $\left(T_{n}^{* *} z\right)(n=1,2, \ldots)$ a Cauchy sequence for each $z$ in $A^{* *}$, there exists a weakly compact operator $T$ such that $\lim _{n \rightarrow \infty} T_{n}^{* *} z=T^{* *} z$ for each $z$ in $A^{* *}$.

Proposition 2.3. Let $A$ be a Banach space with the weak compactness stability property with respect to some non-zero Banach space $X$. Then $A^{*}$ is weakly complete.

Proof. Let $\left(\phi_{n}\right)(n=1,2, \ldots)$ be a weakly Cauchy sequence in $A^{*}$. Then, for each $z$ in $A^{* *}, \lim _{n \rightarrow \infty}\left\langle z, \phi_{n}\right\rangle$ exists. By the uniform boundedness theorem, there exists a bounded linear functional $\psi^{\#}$ on $A^{* *}$ such that $\psi^{\#}(z)=\lim _{n \rightarrow \infty}\left\langle z, \phi_{n}\right\rangle$ for each $z$ in $A^{* *}$.

Since $X$ is a non-zero Banach space it contains a non-zero element $x_{0}$. For each $n$, let $T_{n}: A \mapsto X$ be defined by

$$
T_{n}(a)=\left\langle a, \phi_{n}\right\rangle x_{0}
$$

Then $T_{n}$ has a one-dimensional range and so is (weakly) compact. Furthermore, $T_{n}^{* *}(z)=\left\langle z, \phi_{n}\right\rangle x_{0}$ for each $z$ in $A^{* *}$. It now follows from the weak compactness stability property for $X$ that there exists a weakly compact operator $T$ mapping $A$ into $X$, such that

$$
T^{* *}(z)=\lim _{n \rightarrow \infty} T_{n}^{* *}(z)=\lim _{n \rightarrow \infty}\left\langle z, \phi_{n}\right\rangle x_{0}=\psi^{\#}(z) x_{0} \quad \text { for each } z \text { in } A^{* *}
$$

Since $T$ is weakly compact, then, as remarked in the proof of Theorem $2.1, T^{* *}$ is weak* to weak continuous as a map from $A^{* *}$ to $X$. Thus $\psi^{\#}$ is a weak* continuous linear functional on $A^{* *}$. So, by [2, Theorem V.3.9], $\psi^{\#}$ may be identified with an element of $A^{*}$. Hence $\left(\phi_{n}\right)(n=1,2, \ldots)$ is weakly convergent. Thus $A^{*}$ is weakly complete.

Corollary 2.4. Let $A$ be a Banach space. Then the following conditions are equivalent:

(i) $A^{*}$ is weakly complete;

(ii) A has the weak compactness stability property with respect to some Banach space of non-zero dimension; 
(iii) $A$ has the weak compactness stability property with respect to every Banach space $X$.

Proof. By Theorem 2.1, (i) implies (iii). Trivially (iii) implies (ii). By Proposition 2.3, (ii) implies (i).

\section{Extending Ryan's lemma}

For any Banach space $X$, let $c(X)$ be the Banach space of all (norm) convergent sequences in $X$, equipped with the supremum norm. Those elements of $c(X)$ which are sequences in $X$ converging (in norm) to 0 form a closed subspace which is denoted by $c_{0}(X)$.

For each positive integer $n$, let $T_{n}$ be a bounded linear operator from a Banach space $A$ into a Banach space $X$. Let $\lim T_{n} a$ exist for each $a$ in $A$. Then $\left(T_{n} a\right)(n=1,2, \ldots)$ is a vector in $c(X)$. Let $T_{\infty}$ be the linear map from $A$ into $X$ defined by $T_{\infty} a=\lim T_{n} a$ for each $a$ in $A$. We use $\boldsymbol{T}$ to denote the operator from $A$ to $c(X)$ associated with the sequence $\left(T_{n}\right)(n=1,2, \ldots)$ and defined by $\boldsymbol{T}(a)=\left(T_{n} a\right)(n=1,2, \ldots)$. By applying the uniform boundedness theorem we see that $T_{\infty}$ and $\boldsymbol{T}$ are both bounded linear operators. Conversely, every bounded operator from $A$ into $c(X)$ arises in this way from a sequence of operators from $A$ into $X$.

Let us recall [4] that, for $1 \leqslant p<\infty$ and $X$ an arbitrary Banach space, $l^{p}(X)$ is the Banach space whose points are the sequences $\boldsymbol{x}=\left(x_{n}\right)(n=1,2, \ldots)$ in $X$ for which $\sum_{1}^{\infty}\left\|x_{n}\right\|^{p}<\infty$. The norm of $\boldsymbol{x}$ is defined to be $\left(\sum_{1}^{\infty}\left\|x_{n}\right\|^{p}\right)^{1 / p}$. Also, $l^{\infty}(X)$ is defined to be the Banach space whose points are all bounded sequences in $X$ and where the norm of $\boldsymbol{x}=\left(x_{n}\right)(n=1,2, \ldots)$ is defined to be $\sup \left\{\left\|x_{n}\right\|: 1 \leqslant n\right\}$.

Given $\boldsymbol{\phi}=\left(\phi_{0}, \phi_{1}, \ldots\right)$ in $l^{1}\left(X^{*}\right)$ and $\boldsymbol{x}=\left(x_{n}\right)(n=1,2, \ldots)$ in $c(X)$, let

$$
L_{\boldsymbol{\phi}}(\boldsymbol{x})=\phi_{0}\left(\lim x_{n}\right)+\sum_{n=1}^{\infty}\left\langle x_{n}, \phi_{n}\right\rangle .
$$

Straightforward calculations then show that $L_{\phi}$ is a bounded linear functional on $c(X)$ and its norm is $\sum_{n=0}^{\infty}\left\|\phi_{n}\right\|$. Furthermore, the map $\phi \mapsto L_{\phi}$ can be shown to be a surjective isometry of $l^{1}\left(X^{*}\right)$ onto $c(X)^{*}$.

Then the canonical bilinear form $\langle\cdot, \cdot\rangle$ arising from the dual pair $\left(c(X), c(X)^{*}\right)$, where $l^{1}\left(X^{*}\right)$ and $c(X)^{*}$ are isometrically isomorphic by the map $\phi \mapsto L_{\phi}$ described above, is given by

$$
\left\langle\boldsymbol{x}, L_{\boldsymbol{\phi}}\right\rangle=\left\langle\lim _{n \rightarrow \infty} x_{n}, \phi_{0}\right\rangle+\sum_{n=1}^{\infty}\left\langle x_{n}, \phi_{n}\right\rangle
$$

for each $\boldsymbol{x}=\left(x_{1}, x_{2}, \ldots\right) \in c(X)$ and $\boldsymbol{\phi}=\left(\phi_{0}, \phi_{1}, \ldots\right) \in l^{1}\left(X^{*}\right)$.

It follows from the remarks in [4] that the dual of $l^{1}\left(X^{*}\right)$ can be identified in a natural way with $l^{\infty}\left(X^{* *}\right)$. Thus $c(X)^{* *}$ can be identified with $l^{\infty}\left(X^{* *}\right)$. Let $\natural$ be the canonical embedding of $X$ into $X^{* *}$. Then a sequence $\left(x_{n}\right)(n=1,2, \ldots)$ in $c(X)$ is mapped to $\left(\lim \measuredangle x_{n}, \natural x_{1}, \downarrow x_{2}, \ldots\right)$ in $l^{\infty}\left(X^{* *}\right)$.

Lemma 3.1. Let $\boldsymbol{T}$ be a bounded operator from a Banach space $A$ into $c(X)$ and let $T_{n}(n=1,2, \ldots)$ and $T_{\infty}$ be the operators from $A$ into $X$ associated with $\boldsymbol{T}$ as above. 
Fix $L$ in $c(X)^{*}$. Then let $\phi=\left(\phi_{0}, \phi_{1}, \ldots\right)$ be the corresponding element of $l^{1}\left(X^{*}\right)$. Then, for each $z \in A^{* *}$,

$$
\left\langle\boldsymbol{T}^{* *} z, L\right\rangle=\left\langle T_{\infty}^{* *} z, \phi_{0}\right\rangle+\sum_{n=1}^{\infty}\left\langle T_{n}^{* *} z, \phi_{n}\right\rangle .
$$

Proof. For each $a \in A$,

$$
\langle\boldsymbol{T} a, L\rangle=\left\langle T_{\infty} a, \phi_{0}\right\rangle+\sum_{n=1}^{\infty}\left\langle T_{n} a, \phi_{n}\right\rangle .
$$

Now let $z$ be in the unit ball of $A^{* *}$. Then, by Goldstine's theorem (see above) there is a net $\left(a_{t}\right)$ in the unit ball of $A$ which converges weak ${ }^{*}$ to $z$. Then $\boldsymbol{T} a_{t} \rightarrow \boldsymbol{T}^{* *} z$ in the weak $^{*}$ topology of $c(X)^{* *}$. So $\left\langle\boldsymbol{T} a_{t}, L\right\rangle \rightarrow\left\langle\boldsymbol{T}^{* *} z, L\right\rangle$. Similarly, for each $N$,

$$
\left\langle T_{\infty} a_{t}, \phi_{0}\right\rangle+\sum_{n=1}^{N}\left\langle T_{n} a_{t}, \phi_{n}\right\rangle \rightarrow\left\langle T_{\infty}^{* *} z, \phi_{0}\right\rangle+\sum_{n=1}^{N}\left\langle T_{n}^{* *} z, \phi_{n}\right\rangle .
$$

Choose $\varepsilon>0$. Choose $N$ large enough to ensure that

$$
\|\boldsymbol{T}\| \sum_{n=N+1}^{\infty}\left\|\phi_{n}\right\| \leqslant \varepsilon
$$

Then for any $w$ in the unit ball of $A^{* *}$,

$$
\left|\sum_{n=N+1}^{\infty}\left\langle T_{n}^{* *} w, \phi_{n}\right\rangle\right| \leqslant\|\boldsymbol{T}\| \sum_{n=N+1}^{\infty}\left\|\phi_{n}\right\| \leqslant \varepsilon .
$$

From this it follows by routine arguments that

$$
\left\langle\boldsymbol{T}^{* *} z, L\right\rangle=\left\langle T_{\infty}^{* *} z, \phi_{0}\right\rangle+\sum_{n=1}^{\infty}\left\langle T_{n}^{* *} z, \phi_{n}\right\rangle
$$

We have seen that $c(X)^{* *}$ can be identified with $l^{\infty}\left(X^{* *}\right)$. When this identification is made appropriately, we have the following corollary.

Corollary 3.2. For each $z$ in $A^{* *}$ we have

$$
\boldsymbol{T}^{* *}(z)=\left(T_{\infty}^{* *} z, T_{1}^{* *} z, T_{2}^{* *} z, \ldots, T_{n}^{* *} z, \ldots\right) .
$$

The following lemma was, in essence, proved by Ylinen [6]. For the convenience of the reader, we give a brief proof here as an application of Corollary 3.2.

Lemma 3.3. Let $A$ and $X$ be Banach spaces and let $\boldsymbol{T}$ be a weakly compact operator from $A$ into $c(X)$. Let $\left(T_{n}\right)(n=1,2, \ldots)$ be the sequence of operators from $A$ into $X$ such that $\boldsymbol{T}(a)=\left(T_{n} a\right)(n=1,2, \ldots)$ for each $a$ in $A$. Then each $T_{n}$ is weakly compact. Also, $T_{\infty}$, the pointwise limit of $\left(T_{n}\right)(n=1,2, \ldots)$, is weakly compact. Furthermore, $\lim T_{n}^{* *}(z)=T_{\infty}^{* *} z$ for each $z$ in $A^{* *}$. 
Proof. We recall that the product of a bounded operator and a weakly compact operator is weakly compact. Let $\pi_{n}$ be the canonical projection of $c(X)$ onto the $n$th coordinate. Then $T_{n}=\pi_{n} \boldsymbol{T}$. Hence $T_{n}$ is weakly compact. Let $\pi_{\infty}$ be the operator which maps $\left(a_{1}, a_{2}, \ldots\right)$ in $c(X)$ to $\lim a_{n}$. Then $T_{\infty}=\pi_{\infty} \boldsymbol{T}$ and so is also weakly compact.

Since $\boldsymbol{T}$ is a weakly compact operator from $A$ into $c(X), \boldsymbol{T}^{* *}$ maps $A^{* *}$ into the canonical image of $c(X)$ in the second dual $c(X)^{* *}$. Hence, for every $z \in A^{* *}$, there exists $\boldsymbol{x}=\left(x_{1}, x_{2}, \ldots\right)$ in $c(X)$ such that

$$
\left\langle\left(x_{1}, x_{2}, \ldots\right), L_{\phi}\right\rangle=\left\langle\left(\phi_{0}, \phi_{1}, \phi_{2}, \ldots\right),\left(T_{\infty}^{* *} z, T_{1}^{* *} z, T_{2}^{* *} z, \ldots\right)\right\rangle
$$

for all $\phi=\left(\phi_{0}, \phi_{1}, \phi_{2}, \ldots\right)$.

Now take any $\phi \in X^{*}$ and consider

$$
\phi^{(k)}=\left(\delta_{k, n} \phi\right)_{n=0}^{\infty} \in l^{1}\left(X^{*}\right) \text { for } k=0,1,2, \ldots
$$

Then we have

$$
\left\langle x_{k}, \phi\right\rangle=\left\langle\phi, T_{k}^{* *} z\right\rangle
$$

for all $k \geqslant 1$ and

$$
\left\langle\lim _{k \rightarrow \infty} x_{k}, \phi\right\rangle=\left\langle\phi, T_{\infty}^{* *} z\right\rangle,
$$

that is, $\bigsqcup x_{n}=T_{n}^{* *} z$ for all $n$ and $\lim _{n \rightarrow \infty} \sharp x_{n}=T_{\infty}^{* *} z$. Hence it follows that

$$
\left\|T_{n}^{* *} z-T_{\infty}^{* *} z\right\| \rightarrow 0, \quad n \rightarrow \infty .
$$

Proposition 3.4 (Ryan [4]). Let $A$ and $X$ be Banach spaces. Let $\left(T_{n}\right)(n=1,2, \ldots)$ be a sequence of bounded operators from $A$ into $X$. Let $\left\|T_{n} z\right\| \rightarrow 0$ for each $z$ in $A$. Then $\boldsymbol{T}$ is a weakly compact operator from $A$ into $c_{0}(X)$ if, and only if, each $T_{n}$ is weakly compact and $\left\|T_{n}^{* *} z\right\| \rightarrow 0$ for each $z$ in $A^{* *}$. When $\boldsymbol{T}$ is weakly compact, $\boldsymbol{T}^{* *}(z)=\left(T_{n}^{* *}(z)\right)$ $(n=1,2, \ldots)$ for each $z$ in $A^{* *}$.

Proposition 3.4 is a special case of the following result, which is essentially due to Ylinen $[6]$.

Proposition 3.5. Let $A$ and $X$ be Banach spaces and let $\boldsymbol{T}$ be a bounded operator from $A$ into $c(X)$. Let $\left(T_{n}\right)(n=1,2, \ldots)$ be the sequence of operators from $A$ into $X$ such that $\boldsymbol{T}(a)=\left(T_{n} a\right)(n=1,2, \ldots)$ for each $a$ in $A$ and let $T_{\infty}$ be the operator from $A$ into $X$ which is defined by $T_{\infty} a=\lim T_{n} a$ for each $a \in A$. Then $\boldsymbol{T}$ is weakly compact if and only if the following conditions are satisfied.

(i) For each $n, T_{n}$ is weakly compact.

(ii) For each $z$ in $A^{* *}, \lim T_{n}^{* *}(z)=T_{\infty}^{* *}(z)$.

(iii) The operator $T_{\infty}$ is weakly compact. 
Proof. By Lemma 3.3, when $\boldsymbol{T}$ is weakly compact the three conditions are satisfied.

Now suppose that the conditions are satisfied. So, for each $z$ in $A^{* *}$, condition (iii) implies that $T_{\infty}^{* *} z$ is in $X$ and condition (i) implies that $T_{n}^{* *} z$ is in $X$ for each $n$. Hence, by condition (ii), $\left(T_{\infty}^{* *} z, T_{1}^{* *} z, T_{2}^{* *} z, \ldots, T_{n}^{* *} z, \ldots\right)$ is in the canonical image of $c(X)$ in $c(X)^{* *}$. Hence, by Corollary $3.2, \boldsymbol{T}^{* *}$ maps $A^{* *}$ into $c(X)$. So $\boldsymbol{T}$ is weakly compact.

Theorem 3.6. Let $X$ be any Banach space. Let $A$ be a Banach space whose dual space, $A^{*}$, is weakly complete. Let $\left(T_{n}\right)(n=1,2, \ldots)$ be a sequence of weakly compact operators from $A$ into $X$ such that $\left(T_{n}^{* *}(z)\right)(n=1,2, \ldots)$ is a Cauchy sequence for each $z$ in $A^{* *}$. Then $\boldsymbol{T}$ is a weakly compact operator from $A$ into $c(X)$.

Proof. Because the dual of $A$ is weakly complete, Theorem 2.1 implies the existence of a weakly compact operator $T_{\infty}: A \mapsto X$ such that $T_{n}^{* *}(z) \rightarrow T_{\infty}^{* *}(z)$ for each $z$ in $A^{* *}$. So conditions (i)-(iii) of Proposition 3.5 are satisfied.

Remark. If $A^{*}$ is not weakly complete, then it follows from Proposition 2.3 that we can find a sequence of weakly compact operators, $\left(T_{n}\right)(n=1,2, \ldots)$, each mapping $A$ into $c$, such that $\left(T_{n}^{* *}(z)\right)(n=1,2, \ldots)$ is a convergent sequence for each $z$ in $A^{* *}$ but $T_{\infty}$ is not weakly compact (where $T_{\infty}(a)=\lim T_{n}(a)$ for each $a$ in $A$ ). It then follows from Proposition 3.5 that $\boldsymbol{T}$ is not weakly compact. So the hypothesis that $A^{*}$ is weakly complete is essential for the validity of Theorem 3.6.

\section{References}

1. J. K. Brooks, K. SAitô And J. D. M. Wright, Operator algebras and a theorem of Dieudonné, Rend. Circ. Mat. Palermo 52 (2003), 5-14.

2. N. Dunford And J. T. Schwartz, Linear operators, Volume I (Interscience, New York, 1958).

3. J. M. GutiérRez And I. Villanueva, Extensions of multilinear operators and Banach space properties, Proc. R. Soc. Edinb. A 133 (2003), 549-566.

4. R. A. Ryan, Dunford-Pettis properties, Bull. Polish Acad. Sci. Math. 27 (1979), 373-379.

5. M. TAKESAKI, Theory of operator algebras, Volume I (Springer, 1979).

6. K. YLinen, Weakly compact operators into sequence spaces: a counterexample, Proc. Am. Math. Soc. 133 (2005), 1423-1425. 